\title{
Consequências cardiovasculares na SAOS
}

\author{
Cardiovascular consequences of obstructive sleep apnea syndrome
}

\author{
Geraldo Lorenzi Filho, Pedro Rodrigues Genta, Rodrigo Pinto Pedrosa, \\ Luciano Ferreira Drager, Denis Martinez
}

\begin{abstract}
Resumo
Uma condição clínica muito comum é SAOS, que está associada a várias doenças cardiovasculares, incluindo hipertensão arterial sistêmica, fibrilação atrial e aterosclerose. A associação entre SAOS e doença cardiovascular não é somente uma consequência da sobreposição de fatores de risco, incluindo obesidade, sedentarismo, ser do sexo masculino e ter idade maior. Existem evidências crescentes de que SAOS contribui de forma independente para o aparecimento e a progressão de várias doenças cardiovasculares. Os mecanismos pelos quais SAOS pode afetar o sistema cardiovascular são múltiplos e incluem a ativação do sistema nervoso simpático, inflamação sistêmica, resistência a insulina e geração de estresse oxidativo. Existem evidências que o tratamento de SAOS com CPAP pode reduzir a pressão arterial, sinais precoces de aterosclerose, risco de recorrência de fibrilação atrial e mortalidade, principalmente por acidente vascular cerebral e infarto agudo do miocárdio, em pacientes com SAOS grave.
\end{abstract}

Descritores: Apneia do sono tipo obstrutiva/terapia; Doenças cardiovasculares; Pressão positiva contínua nas vias aéreas.

\begin{abstract}
Obstructive sleep apnea syndrome (OSAS) is a common condition associated with various cardiovascular diseases, including systemic arterial hypertension, atrial fibrillation, and atherosclerosis. The association between OSAS and cardiovascular disease has been related to the overlapping of risk factors, including obesity, having a sedentary lifestyle, being male, and being older. However, there is mounting evidence that OSAS can also independently contribute to the development and progression of various cardiovascular diseases. The mechanisms by which OSAS can affect the cardiovascular system are multiple, including the activation of the sympathetic nervous system, systemic inflammation, insulin resistance, and oxidative stress. There is also evidence that the treatment of OSAS with CPAP can reduce arterial blood pressure, early signs of atherosclerosis, the risk of atrial fibrillation recurrence, and mortality (principally mortality due to stroke or acute myocardial infarction) in patients with severe OSAS.
\end{abstract}

Keywords: Sleep apnea, obstructive/therapy; Cardiovascular diseases; Continuous positive airway pressure.

\section{Introdução}

Desde as descrições iniciais, foi reconhecido que SAOS está associada a várias doenças cardiovasculares. Foi inicialmente descrita uma alta prevalência de arritmia cardíaca noturna, cor pulmonale e hipertensão arterial pulmonar e sistêmica entre os pacientes com SAOS.(1,2) Essa associação pode ser em função da sobreposição de fatores de risco comuns, tanto para SAOS, como para doença cardiovascular (obesidade, homens de meia-idade e mulheres após a menopausa, sedentarismo e tabagismo). De fato, a prevalência de SAOS entre pacientes com diagnóstico de doença cardiovascular já estabelecida é alarmante. Por exemplo, entre pacientes com hipertensão e hipertensão refratária, a prevalência de SAOS gira em torno de $30 \%$ e $70 \%$, respectivamente. ${ }^{(3,4)}$ Entre pacientes com fibrilação atrial, a prevalência estimada de SAOS é de 50\%.(5) É importante destacar que a maior parte dos pacientes com doença cardiovascular permanece sem diagnóstico. Esse fato pode ser explicado por um baixo grau de suspeita diagnóstica de SAOS, além da dificuldade de acesso à polissonografia noturna. Outro fato que tem chamado a atenção é que os pacientes com SAOS advindos da população de pacientes com doença cardiovascular frequentemente não se queixam de sonolência excessiva diurna. ${ }^{(3,6)}$ As causas para essa particularidade são ainda desconhecidas, mas certamente contribuem para dificultar a suspeita clínica de SAOS. Somente o reconhecimento pela comunidade médica de que SAOS é comum entre os pacientes com doença cardiovascular já seria de extrema importância. No entanto, essa breve revisão vai explorar as evidências crescentes que apontam 
para uma relação causal entre SAOS e doença cardiovascular. Essa síndrome pode contribuir para o estabelecimento ou piora de várias doenças cardiovasculares, o que torna o cenário de falta de reconhecimento e de tratamento adequado de SAOS ainda mais dramático.

Exploraremos inicialmente os mecanismos fisiopatológicos que justificam uma relação causal entre SAOS e doença cardiovascular. Uma linha de evidência apontando para a associação independente entre SAOS e doença cardiovascular advém de estudos epidemiológicos. Vários trabalhos populacionais demonstraram uma associação independente entre SAOS e hipertensão arterial sistêmica, insuficiência coronariana, acidente vascular cerebral e insuficiência cardíaca congestiva. ${ }^{(7-9)}$ Finalmente, a relação entre SAOS e algumas das principais doenças cardiovasculares serão exploradas.

\section{Fisiopatologia da ligação entre SAOS e doença cardiovascular}

Os mecanismos primários de SAOS que são deletérias ao sistema cardiovascular são os seguintes: hipóxia intermitente, microdespertares e geração de pressão intratorácica negativa durante os eventos respiratórios obstrutivos (apneias ou hipopneias) associados a aumento de pressão arterial (Figura 1).(1) Um conceito importante é que as complicações advindas dos eventos respiratórios não se restringem ao período noturno, mas podem se estender ao longo do dia. Uma lista crescente de alterações bioquímicas, inflamatórias, metabólicas e vasculares, associadas de forma independente a SAOS, foram descritas e incluem aumento da atividade simpática, aumento nos níveis plasmáticos de proteína $\mathrm{C}$ reativa, de citocinas e de fibrinogênio, resistência à insulina, leptina e a produtos derivados de estresse oxidativo e disfunção endotelial. ${ }^{(10-12)}$

Uma dificuldade constante é comprovar uma relação causal entre SAOS e doença cardiovascular, visto que vários fatores de confusão (em particular a obesidade) são frequentes nesses pacientes. Contribuem para demonstrar a relação causal entre SAOS e doença cardiovascular os estudos que demonstram que o tratamento de SAOS com CPAP reduz ou normaliza várias dessas disfunções, incluindo redução da atividade simpática, dos níveis de

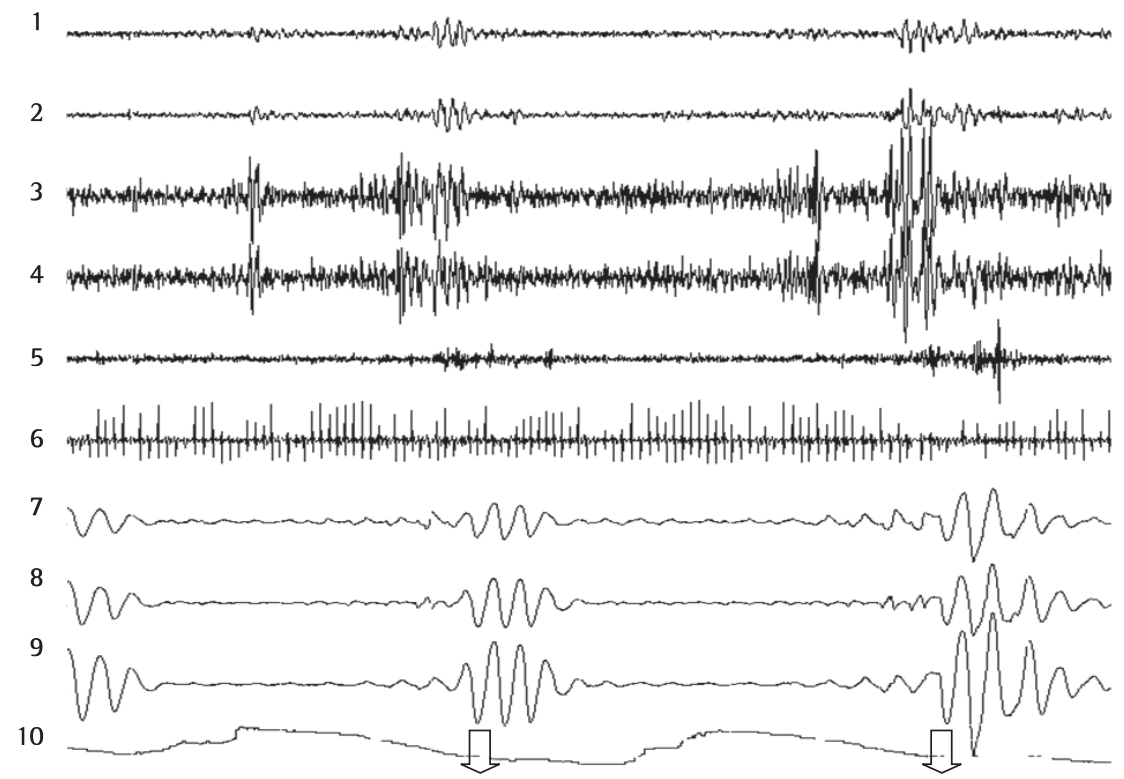

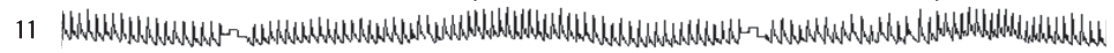

Figura 1 - Traçado polissonográfico padrão, com adição do canal 11, registro de PA por Finapress, batimento a batimento. Note-se o aumento de PA e de frequência cardíaca ao término de cada apneia (setas). Canais 1 e 2, eletrooculograma; 3 e 4, eletroencefalograma; 5, eletromiograma; 6 , eletrocardiograma; 7 , movimento torácico; 8, movimento abdominal; 9, volume corrente; 10, saturação de oxigênio arterial. 
proteína C reativa e da resistência a insulina, além de melhora da disfunção endotelial. ${ }^{(10,11,13)}$

\section{Hipertensão arterial sistêmica}

A relação mais bem estudada entre SAOS e doença cardiovascular é a sua relação com hipertensão arterial sistêmica. Os potenciais mecanismos que ligam SAOS a hipertensão arterial sistêmica são múltiplos e incluem ativação simpática, diminuição da sensibilidade dos barorreceptores, hiperresponsividade vascular e alteração no metabolismo do sal e água. Pacientes com SAOS apresentam aumentos cíclicos da pressão arterial associados aos episódios de apneia. Os pacientes com SAOS frequentemente não apresentam o descenso noturno da pressão arterial e são considerados como non-dippers. A confirmação da relação causal entre SAOS e hipertensão arterial sistêmica é baseada em estudos experimentais com vários modelos animais, estudos epidemiológicos e estudos de tratamento de SAOS. 0 estudo mais citado da literatura é advindo de uma coorte em Wisconsin, EUA, no qual foram avaliados 709 indivíduos através de estudo polissonográfico. ${ }^{(7)}$ Após um seguimento de quatro anos, mostrou-se uma associação causal entre a presença de SAOS na avaliação inicial e o surgimento de hipertensão arterial sistêmica no seguimento. Nesse mesmo estudo, os autores observaram uma relação dose-resposta entre a gravidade de SAOS e o risco de aparecimento de hipertensão arterial. De acordo com o sétimo relatório do Joint National Committee on Prevention, Detection, Evaluation and Treatment of High Blood Pressure, SAOS é a primeira de uma lista de causas identificáveis de hipertensão arterial sistêmica. ${ }^{(14)}$

Diversos estudos avaliaram o efeito do tratamento de SAOS no controle da pressão arterial. No entanto, apenas dois ensaios clínicos avaliaram uma população com predomínio de pacientes hipertensos descontrolados e apresentaram resultados opostos. Em um estudo de dois meses de duração envolvendo pacientes com sonolência diurna e SAOS, randomizados para CPAP ou sham CPAP (isto é, CPAP com pressões mínimas sem efeito terapêutico), houve uma redução de $10 \mathrm{mmHg}$ na pressão arterial sistólica e diastólica de $24 \mathrm{~h}$ no grupo CPAP. ${ }^{(15)}$ Já em outro estudo mais curto e com um número menor de participantes, em que os pacientes sem sonolência diurna fizeram um cross-over para o uso de CPAP, não houve redução da pressão arterial com o uso de CPAP. ${ }^{(16)}$

Uma recente meta-análise avaliou 818 pacientes e demonstrou uma redução da pressão arterial de apenas $2,46 \mathrm{mmHg}$ na pressão arterial sistólica e de $1,83 \mathrm{mmHg}$ na pressão arterial diastólica após o uso de CPAP. (17) Entretanto, a escolha dos trabalhos incluídos nessa meta-análise é sujeita a críticas (envolveu estudos com indivíduos normotensos e pacientes com insuficiência cardíaca), o que pode ter contribuído para o resultado modesto da queda da pressão arterial. Além disso, a redução da pressão arterial pode não ser o único ou mesmo o maior efeito do uso de CPAP no sistema cardiovascular, já que têm sido descritos outros benefícios cardiovasculares, conforme descrito anteriormente.

\section{Arritmias cardíacas}

Existem evidências de que SAOS está associada de forma independente com fibrilação atrial e batimentos ventriculares ectópicos. Por exemplo, em um estudo transversal com 2.911 homens idosos que realizaram polissonografia, encontrou-se uma associação entre a gravidade de apneia com fibrilação atrial e batimentos ventriculares ectópicos. ${ }^{(18)}$ Mais importante, existem evidências de que, após a reversão de fibrilação atrial, SAOS esteja associada a um aumento do risco de recorrência de fibrilação atrial após um ano e que o tratamento da SAOS com CPAP reduza esse risco. (5) Em um estudo recente com 73 indivíduos com e sem SAOS pareados para fatores de confusão, mostrou-se que SAOS estava associada ao aumento do diâmetro do átrio esquerdo que, por sua vez, se correlacionou com o aumento da rigidez da aorta. ${ }^{(19)}$ Considerando-se que o tamanho do átrio esquerdo é um fator de risco para a fibrilação atrial, esse estudo sugere que o aumento da rigidez da aorta observado em pacientes com SAOS possa contribuir para o remodelamento atrial devido a um aumento da pós-carga do ventrículo esquerdo.

\section{Aterosclerose, doença coronariana e doença cerebrovascular}

Há uma associação entre SAOS grave não tratada e um aumento da mortalidade cardiovascular causada por acidente vascular 
cerebral e infarto agudo do miocárdio. ${ }^{(20,21)}$ Nesses estudos, sugeriu-se que SAOS contribui para o desenvolvimento de aterosclerose. Essa hipótese é reforçada pela demonstração que pacientes com SAOS sem comorbidades, quando comparados com controles adequados, apresentam vários marcadores de aterosclerose alterados, incluindo maior rigidez arterial e aumento da espessura intima média da carótida. ${ }^{(22,23)}$ A aterosclerose observada na prática pode ser ainda pior, pois soma-se o efeito de SAOS com o de várias comorbidades frequentemente presentes, incluindo a hipertensão arterial sistêmica e a síndrome metabólica. ${ }^{(24,25)}$ De forma semelhante, a calcificação coronariana tem sido atribuída de forma independente à marcadores de gravidade de SAOS em pacientes com comorbidades. ${ }^{(26)}$ Uma evidência recente do nosso grupo suporta a hipótese de que mais do que uma associação, SAOS parece ser uma causa para a ocorrência e a progressão da aterosclerose. Em um estudo envolvendo pacientes com SAOS grave randomizados para tratamento com CPAP e conduta expectante, quatro meses de tratamento foram suficientes para reduzir marcadores de aterosclerose, incluindo a espessura intima média da carótida e a rigidez arterial, medida pela velocidade de onda de pulso carótideofemoral. ${ }^{(23)}$

\section{Referências}

1. Lorenzi-Filho G, Bradley TD. Cardiac function in sleep apnea. In: Pack Al. Sleep Apnea: Pathogenesis, Diagnosis, and Treatment. New York: M Dekker; 2002. p. 377-410.

2. Logan AG, Perlikowski SM, Mente A, Tisler A, Tkacova $R$, Niroumand $M$, et al. High prevalence of unrecognized sleep apnoea in drug-resistant hypertension. J Hypertens. 2001;19(12):2271-7.

3. Gus M, Gonçalves SC, Martinez D, de Abreu Silva EO, Moreira LB, Fuchs SC, et al. Risk for Obstructive Sleep Apnea by Berlin Questionnaire, but not daytime sleepiness, is associated with resistant hypertension: a case-control study. Am J Hypertens. 2008;21(7):832-5.

4. Gonçalves SC, Martinez D, Gus M, de Abreu-Silva EO, Bertoluci C, Dutra 1, et al. Obstructive sleep apnea and resistant hypertension: a case-control study. Chest. 2007;132(6):1858-62.

5. Caples SM, Somers VK. Sleep-disordered breathing and atrial fibrillation. Prog Cardiovasc Dis. 2009;51(5):411-5.

6. Drager LF, Queiroz EL, Lopes HF, Genta PR, Krieger EM, Lorenzi-Filho G. Obstructive sleep apnea is highly prevalent and correlates with impaired glycemic control in consecutive patients with the metabolic syndrome. J Cardiometab Syndr. 2009;4(2):89-95.7. Peppard PE, Young T, Palta M, Skatrud J. Prospective study of the association between sleep-disordered breathing and hypertension. N Engl J Med. 2000;342(19):1378-84.

8. Shahar E, Whitney CW, Redline S, Lee ET, Newman AB, Javier Nieto F, et al. Sleep-disordered breathing and cardiovascular disease: cross-sectional results of the Sleep Heart Health Study. Am J Respir Crit Care Med. 2001;163(1):19-25.

9. Peker Y, Hedner J, Norum J, Kraiczi H, Carlson J. Increased incidence of cardiovascular disease in middle-aged men with obstructive sleep apnea: a 7-year follow-up. Am J Respir Crit Care Med. 2002;166(2):159-65.

10. Shamsuzzaman AS, Somers VK. Fibrinogen, stroke, and obstructive sleep apnea: an evolving paradigm of cardiovascular risk. Am J Respir Crit Care Med. 2000;162(6):2018-20.

11. Ip MS, Lam B, Ng MM, Lam WK, Tsang KW, Lam KS. Obstructive sleep apnea is independently associated with insulin resistance. Am J Respir Crit Care Med. 2002;165(5):670-6.

12. Minoguchi K, Yokoe T, Tazaki T, Minoguchi H, Tanaka A, Oda N, et al. Increased carotid intima-media thickness and serum inflammatory markers in obstructive sleep apnea. Am J Respir Crit Care Med. 2005;172(5):62530.

13. Kato M, Adachi T, Koshino Y, Somers VK. Obstructive sleep apnea and cardiovascular disease. Circ J. 2009;73(8):1363-70.

14. Chobanian AV, Bakris GL, Black HR, Cushman WC, Green LA, Izzo JL Jr, et al. The Seventh Report of the Joint National Committee on Prevention, Detection, Evaluation, and Treatment of High Blood Pressure: the JNC 7 report. JAMA. 2003;289(19):2560-72. Erratum in: JAMA. 2003;290(2):197.

15. Mills PJ, Kennedy BP, Loredo JS, Dimsdale JE, Ziegler MG. Effects of nasal continuous positive airway pressure and oxygen supplementation on norepinephrine kinetics and cardiovascular responses in obstructive sleep apnea. J Appl Physiol. 2006;100(1):343-8.

16. Robinson GV, Smith DM, Langford BA, Davies RJ, Stradling JR. Continuous positive airway pressure does not reduce blood pressure in nonsleepy hypertensive OSA patients. Eur Respir J. 2006;27(6):1229-35.

17. Bazzano LA, Khan Z, Reynolds K, He J. Effect of nocturnal nasal continuous positive airway pressure on blood pressure in obstructive sleep apnea. Hypertension. 2007;50(2):417-23.

18. Mehra R, Stone KL, Varosy PD, Hoffman AR, Marcus GM, Blackwell T, et al. Nocturnal Arrhythmias across a spectrum of obstructive and central sleep-disordered breathing in older men: outcomes of sleep disorders in older men (MrOS sleep) study. Arch Intern Med. 2009;169(12):1147-55.

19. Drager LF, Bortolotto LA, Pedrosa RP, Krieger EM, Lorenzi-Filho G. Left atrial diameter is independently associated with arterial stiffness in patients with obstructive sleep apnea: Potential implications for atrial fibrillation. Int J Cardiol. Epub 2009.

20. Marshall NS, Wong KK, Liu PY, Cullen SR, Knuiman MW, Grunstein RR. Sleep apnea as an independent risk factor for all-cause mortality: the Busselton Health Study. Sleep. 2008;31(8):1079-85.

21. Marin JM, Carrizo SJ, Vicente E, Agusti AG. Long-term cardiovascular outcomes in men with obstructive sleep apnoea-hypopnoea with or without treatment with 
continuous positive airway pressure: an observational study. Lancet. 2005;365(9464):1046-53.

22. Drager LF, Bortolotto LA, Lorenzi MC, Figueiredo AC, Krieger EM, Lorenzi-Filho G. Early signs of atherosclerosis in obstructive sleep apnea. Am J Respir Crit Care Med. 2005;172(5):613-8.

23. Drager LF, Bortolotto LA, Figueiredo AC, Krieger EM, Lorenzi GF. Effects of continuous positive airway pressure on early signs of atherosclerosis in obstructive sleep apnea. Am J Respir Crit Care Med. 2007;176(7):706-12.

24. Drager LF, Bortolotto LA, Krieger EM, Lorenzi-Filho G. Additive effects of obstructive sleep apnea and hypertension on early markers of carotid atherosclerosis. Hypertension. 2009;53(1):64-9.

25. Drager LF, Bortolotto LA, Maki-Nunes C, Trombetta IC, Alves MJ, Fraga RF, et al. The incremental role of obstructive sleep apnoea on markers of atherosclerosis in patients with metabolic syndrome. Atherosclerosis. 2010;208(2):490-5.

26. Sorajja D, Gami AS, Somers VK, Behrenbeck TR, GarciaTouchard A, Lopez-Jimenez F. Independent association between obstructive sleep apnea and subclinical coronary artery disease. Chest. 2008;133(4):927-33.

\section{Sobre os autores}

\section{Geraldo Lorenzi Filho}

Professor Livre-Docente. Disciplina de Pneumologia, Faculdade de Medicina, Universidade de São Paulo, São Paulo (SP) Brasil.

\section{Pedro Rodrigues Genta}

Médico Pneumologista. Laboratório do Sono, Disciplina de Pneumologia, Faculdade de Medicina, Universidade de São Paulo, São Paulo (SP) Brasil.

\section{Rodrigo Pinto Pedrosa}

Médico Plantonista. Hospital Universitário Oswaldo Cruz, Universidade de Pernambuco, Recife (PE) Brasil.

\section{Luciano Ferreira Drager}

Médico Assistente. Unidade de Hipertensão, Instituto do Coração, Hospital das Clínicas, Faculdade de Medicina, Universidade de São Paulo, São Paulo (SP) Brasil.

\section{Denis Martinez}

Professor Associado 1l. Faculdade de Medicina, Universidade Federal do Rio Grande do Sul, Porto Alegre (RS) Brasil. E-mail para contato: geraldo.lorenzi@incor.usp.br (G. Lorenzi-Filho) ou sono@sono.com.br (D. Martinez) 